\title{
Subscapularis- and deltoid-sparing vs traditional deltopectoral approach in reverse shoulder arthroplasty: a prospective case-control study
}

\author{
Alexandre Lädermann ${ }^{1,2,3^{*}}$ (D), Patrick Joel Denard ${ }^{4,5}$, Jérome Tirefort ${ }^{3}$, Philippe Collin ${ }^{6}$, Alexandra Nowak ${ }^{1}$
} and Adrien Jean-Pierre Schwitzguebel ${ }^{1}$

\begin{abstract}
Background: With the growth of reverse shoulder arthroplasty (RSA), it is becoming increasingly necessary to establish the most cost-effective methods for the procedure. The surgical approach is one factor that may influence the cost and outcome of RSA. The purpose of this study was to compare the clinical results of a subscapularis- and deltoid-sparing (SSCS) approach to a traditional deltopectoral (TDP) approach for RSA. The hypothesis was that the SSCS approach would be associated with decreased length of stay (LOS), equal complication rate, and better short-term outcomes compared to the TDP approach.

Methods: A prospective evaluation was performed on patients undergoing RSA over a 2-year period. A deltopectoral incision was used followed by either an SSCS approach or a traditional tenotomy of the subscapularis (TDP). LOS, adverse events, physical therapy utilization, and patient satisfaction were collected in the 12 months following RSA.

Results: LOS was shorter with the SSCS approach compared to the TDP approach (from $8.2 \pm 6.4$ days to $15.2 \pm 11$. 9 days; $P=0.04)$. At 3 months postoperative, the single assessment numeric evaluation score $(80 \pm 11 \%$ vs $70 \pm 6 \%$; $P=0.04)$ and active elevation ( $130 \pm 22^{\circ}$ vs $\left.109 \pm 24^{\circ} ; P=0.01\right)$ were higher in the SSCS group. The SSCS approach resulted in a net cost savings of $\$ 5900$ per patient. Postoperative physical therapy, pain levels, and patient satisfaction were comparable in both groups. No immediate intraoperative complications were noted.
\end{abstract}

Conclusion: Using a SSCS approach is an option for patients requiring RSA. Overall LOS is minimized compared to a TDP approach with subscapularis tenotomy. The SSCS approach may provide substantial healthcare cost savings, without increasing complication rate or decreasing patient satisfaction.

Keywords: Shoulder, Reverse shoulder arthroplasty, Length of stay, Deltopectoral approach, Subscapularis sparing, Approach, Cost-effectiveness, Results

\section{Background}

The use of reverse shoulder arthroplasty (RSA) has increased substantially in recent years [1]. While the introduction of RSA has provided a solution for several previously untreatable conditions, as with most technological advancements, this has led to increased healthcare

\footnotetext{
* Correspondence: alexandre.laedermann@gmail.com

'Division of Orthopaedics and Trauma Surgery, La Tour Hospital, Rue J.-D. Maillard 3, 1217 Meyrin, Switzerland

${ }^{2}$ Faculty of Medicine, University of Geneva, Rue Michel-Servet 1, 1211 Geneva 4, Switzerland

Full list of author information is available at the end of the article
}

utilization and cost. Concurrently, from a macroscopic perspective, there has been growing interest within health systems to identify the most valuable or cost-effective treatments.

The bundled payment initiative has brought attention to examining multiple aspects of cost in the entire phase of care. In addition to implant cost, potential areas of cost savings include length of stay (LOS), complication and readmission rate, and postoperative rehabilitation center or physical therapy utilization. The impact of surgical approach for RSA on the aforementioned factors 
has not been previously studied. The most common surgical approach for RSA is a deltopectoral incision that includes a tenotomy or peel of the subscapularis to gain access to the glenohumeral joint. Recently, an approach which uses a deltopectoral incision but spares the subscapularis has been reported with good short-term clinical results [2]. Since this approach is subscapularis and deltoid sparing (SSCS), immediate active range of motion (ROM) without immobilization is allowed [2]. This fast-track rehabilitation protocol may therefore lead to cost savings.

The purpose of this study was to compare the clinical results of the SSCS and the TDP for RSA. The hypothesis was that the SSCS approach would be associated with decreased LOS, equal complication rate, and better short-term outcomes compared to a traditional deltopectoral (TDP) approach.

\section{Methods}

\section{Patient selection}

Between May 2013 and June 2015, all patients who had a primary RSA performed by one author (A.L.) with minimum follow-up of 3 months were considered potentially eligible for inclusion in this prospective casecontrol study that estimated the cost savings of a TDP approach compared to the SSCS approach. Patients with fractures, previous infection, shoulder malignancy, and revision surgery were excluded. Forty-three patients were considered potentially eligible for the study. Among them, five were excluded for revision shoulder arthroplasty, one for shoulder malignancy, and two for previous glenohumeral septic arthritis. Thus, there were 35 patients (35 RSAs) that met the study criteria. There were 18 patients in the TDP group and 17 patients in the SSCS group (Fig. 1). The study protocol was approved by the hospital ethics committee (AMG: 12-26), and all patients gave informed written consent.

\section{Surgical technique}

All patients had general anesthesia with muscle relaxants used to facilitate glenoid exposure. Prior to skin incision, prophylactic intravenous antibiotics (cefazolin) were administered. In all cases, a deltopectoral incision was used [3]. The two approaches vary at the point of addressing the subscapularis tendon. If the subscapularis was torn, the remaining subscapularis and/or capsular tissue was tenotomized to gain access to the glenohumeral joint [4]. Conversely, if the subscapularis was intact, a SSCS approach was utilized as previously described [2]. For both approaches, the humeral head was cut with $20^{\circ}$ of retroversion [5-7]. A circular baseplate (Aequalis Reversed; Tornier, Montbonnot, France) was implanted at the inferior edge of the glenoid. The glenosphere was usually eccentric to limit friction-type impingement in

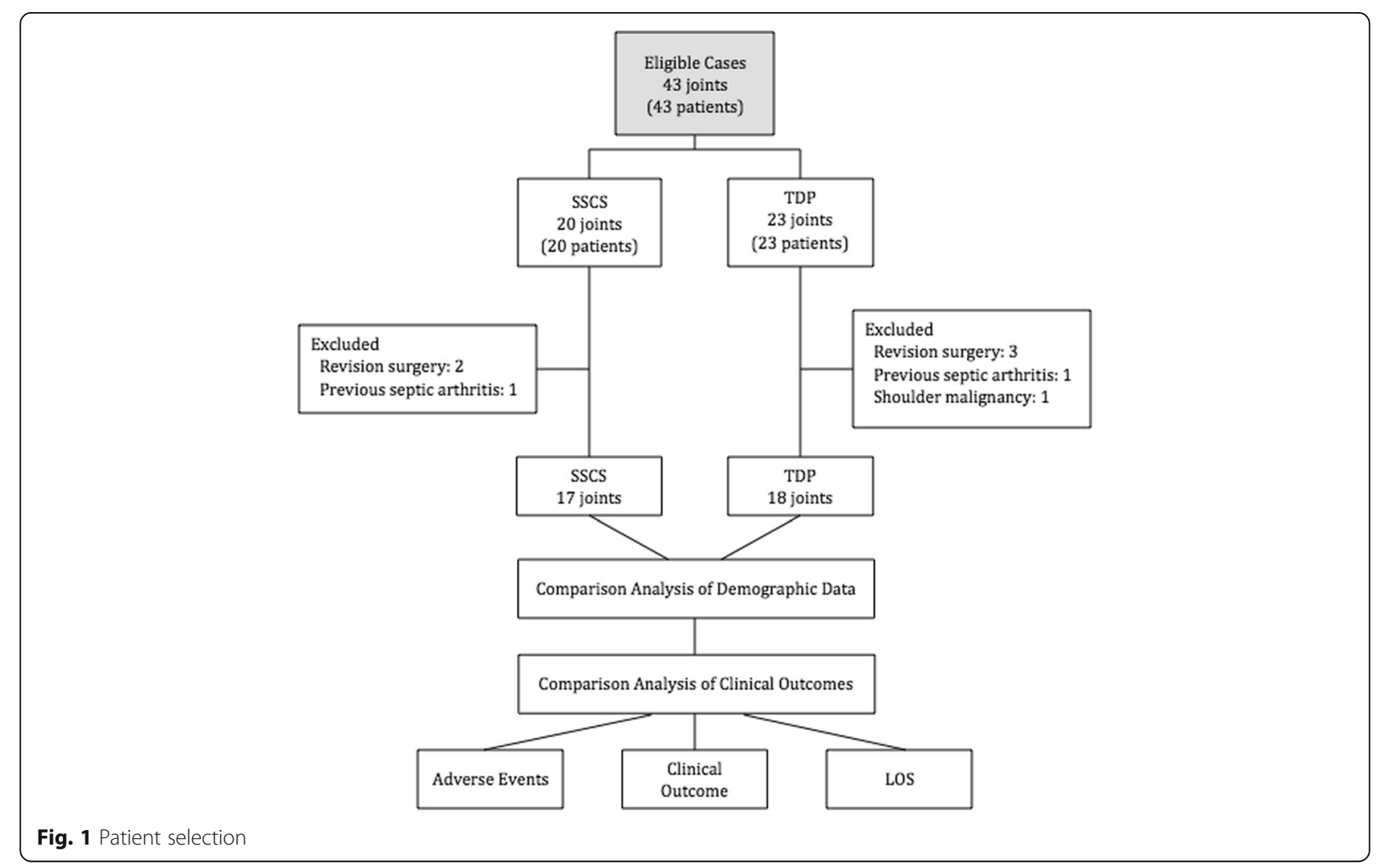


adduction, extension, and external rotation [8]. An onlay humeral stem with a final humeral inclination of $145^{\circ}$ and an eccentric humeral plate was implanted. The eccentric infero-medial position was always used to limit arm lengthening and to maximize lateralization [9]. After closure of the incision, $160 \mathrm{mg}$ of gentamicin mixed in $20 \mathrm{~mL}$ of saline was injected into the glenohumeral joint [10].

\section{Postoperative rehabilitation}

In the case of a TDP approach, a standardized rehabilitation protocol was followed [11]. Patients were placed in a sling for 4 weeks. Passive ROM was initiated immediately, and active motion was allowed at 4 weeks. Strengthening was allowed at 8 weeks. With the SSCS approach, immediate active ROM was allowed with a sling for comfort only during the first few postoperative days [2] and strengthening was allowed at 6 weeks.

\section{Baseline characteristics and study variables}

Baseline clinical characteristics extracted from the prospective database included age, sex, dominant hand, initial diagnosis (Hamada 1 to 2, Hamada 3 to 5, dislocation arthropathy, post-traumatic), previous shoulder surgeries, prior deltoid or subscapularis insufficiency, and baseline functional outcome and ROM. Baseline characteristics are summarized in Table 1.

The primary outcome was LOS, including hospitalization and rehabilitation or post acute care. LOS during hospitalization was determined by the ability of the patient to return home. If unable, rehabilitation or post acute care was prescribed until the patient was able to independently return home. All costs were expressed in US dollars and estimated by adding the costs of the immediate postoperative hospital stay and rehabilitation stay. At our institution, the average cost of a hospital stay per night is approximately $\$ 1500$ and the cost of a rehabilitation stay per night is approximately $\$ 647$. Implant costs were excluded since we used the same implant in all cases and were not evaluating implant costs.

Secondary outcomes were adverse events (readmission and complication), number of postoperative physical therapy sessions, and clinical outcome at 3 months in terms of pain (visual analogue scale (VAS)), functional outcome (single assessment numeric evaluation (SANE)), and ROM in elevation, external rotation, and internal rotation) and at 12 months with Constant score [12]. Preoperative outcomes are summarized in Table 2.

\section{Statistical analysis}

Statistical analysis was performed with R v3.1.2 Portable (Free Software Foundation Inc, Vienna, Austria). Basic descriptive statistics (mean and percentages) were used for baseline clinical parameters and functional evaluation (VAS, SANE, and ROM). Clinical parameters of interest were compared between SSCS and TDS approach with two-tailed Student's $t$ or chi-squared test, when appropriate. Level of significance was set at $P<0.05$.

\section{Results}

There were no statistically significant differences between the two groups at baseline (Tables 1 and 2).

With the SSCS approach, the total length of stay was significantly shorter compared to the TDP approach. Hospitalization and rehabilitation stay costs were lower in the SSCS approach compared to the TDP approach (Table 3). There were no statistically significant differences between groups with respect to the number of physical therapy sessions. The SSCS approach was associated with a better functional outcome at 3 months in regard to SANE score and arm elevation. There was no statistically significant difference between the two groups in postoperative pain or range of internal and external rotation at 3 months postoperative (Table 4) and in Constant score at 1 year $(68.1 \pm 15.6$ with SSCS approach vs $77.3 \pm 12.9$ with TDP approach; $P=0.07$, respectively).

Table 1 Baseline patient characteristics

\begin{tabular}{lllll}
\hline & All prosthesis $(N=35)$ & SSCS approach $(N=17)$ & TDP approach $(N=18)$ & $P$ \\
\hline DRG insurance coverage & 14 & 9 & 5 & 3 \\
Failed cuff repair & 3 & 0 & 4 & 0.13 \\
Cuff tear arthropathy Hamada 1-2 & 14 & 10 & 5 & 0.08 \\
Cuff tear arthropathy Hamada 3-5 & 10 & 5 & 6 & 0.91 \\
Malunion & 8 & 2 & $78 \pm 8$ & 0.13 \\
Age & $78 \pm 7$ & $78 \pm 7$ & $4(22 \%)$ & 0.82 \\
Sex (male) & $8(23 \%)$ & $4(24 \%)$ & $11(61 \%)$ & 1 \\
Dominant arm & $18(51 \%)$ & $7(41 \%)$ & $15(94 \%)$ & 0.4 \\
Previous surgeries & $20(61 \%)$ & $5(29 \%)$ & & 0.23 \\
\hline
\end{tabular}

DRG diagnosis-related group, TDP traditional deltopectoral, SSCS subscapularis and deltoid sparing 
Table 2 Preoperative outcomes

\begin{tabular}{lllll}
\hline & $\begin{array}{l}\text { All prosthesis } \\
(N=35)\end{array}$ & $\begin{array}{l}\text { SSCS approach } \\
(N=17)\end{array}$ & $\begin{array}{l}\text { TDP approach } \\
(N=18)\end{array}$ & $P$ \\
\hline Pain VAS & $6.9 \pm 2.3$ & $6.9 \pm 1.9$ & $6.8 \pm 2.7$ & 0.84 \\
SANE & $32 \pm 19$ & $37 \pm 14$ & $27 \pm 22$ & 0.12 \\
Forward elevation & $95 \pm 50$ & $111 \pm 58$ & $75 \pm 31$ & 0.04 \\
ER & $19 \pm 20$ & $20 \pm 21$ & $19 \pm 18$ & 0.9 \\
$\begin{array}{l}\text { IR (median spinal } \\
\text { height) }\end{array}$ & $L 4$ & $L 1$ & Sacrum & 0.27 \\
\hline
\end{tabular}

$E R$ external rotation, IR internal rotation, TDP traditional deltopectoral, SANE single shoulder numeric assessment, SSCS subscapularis and deltoid sparing, VAS visual analogue scale

During the follow-up period of $18 \pm 11$ months (range, 12 to 46 months), only one patient had a complication. This patient had a TDP approach and suffered a prosthetic dislocation 6 weeks postoperatively, which has been successfully managed with closed reduction. The same patient also experienced an acromial stress fracture that was managed conservatively. No subscapularis avulsion or iatrogenic tuberosity fracture was observed due to retraction during the SSCS approach.

\section{Discussion}

The results of the current study support the hypothesis that the SSCS approach is associated with lower cost and equal complication compared to a TDP approach.

It is notable that the population is aging and older patients are the most likely to benefit from RSA [13]. However, the average hospital cost for shoulder arthroplasty is estimated to be $\$ 17,000$ [14]. Consequently, it is important to find a solution to reduce the overall costs to provide continued access to RSA. LOS has recently been analyzed after shoulder arthroplasty in women, seniors, and comorbidity patients, with insurance coverage and diagnosis significantly contributing to increase in LOS $[13,15,16]$. In addition, hospital volume and surgeon experience have been associated with a lower LOS and cost compared to lower volume facilities and surgeons [17]. The current study examines an additional variable-that of surgical approach-which may affect cost. After controlling for preoperative and surgical variables, utilization of a SSCS approach compared to the current standard of a TDP approach for RSA resulted in an economic savings of $\$ 5881$, corresponding to an average
Table 4 Clinical outcome evaluated at 3 months post-surgery

\begin{tabular}{lllll}
\hline & $\begin{array}{l}\text { All prosthesis } \\
(N=35)\end{array}$ & $\begin{array}{l}\text { SSCS } \\
(N=17)\end{array}$ & $\begin{array}{l}\text { TDP } \\
(N=18)\end{array}$ & P value \\
\hline Pain VAS & $1.2 \pm 1.4$ & $1.2 \pm 1.5$ & $1.2 \pm 1.4$ & 0.89 \\
SANE & $75 \pm 15$ & $80 \pm 11$ & $70 \pm 16$ & 0.04 \\
Forward elevation & $119 \pm 25$ & $130 \pm 22$ & $109 \pm 24$ & 0.01 \\
ER & $20 \pm 24$ & $25 \pm 27$ & $15 \pm 21$ & 0.29 \\
IR (median spinal level) & L4 & L1 & L4 & 0.27
\end{tabular}

ER external rotation, IR internal rotation, TDP traditional deltopectoral, SANE Single shoulder numeric assessment, SSCS subscapularis and deltoid sparing, VAS visual analogue scale

LOS of 7 days. By decreasing LOS and allowing earlier mobilization, such an approach may also help lower hospital-acquired infection rates [18], decrease risk factors for readmission [19], and improve patient satisfaction [20].

In addition to cost savings, the SSCS approach group was also significantly associated with a better functional outcome at 3 months compared to the TDP approach. At least four reasons could explain these differences. First, the subscapularis plays a crucial role in anterior elevation. Collin et al. previously demonstrated that the subscapularis is the most important rotator cuff muscle for elevation in native shoulders [21]. Although the RSA design partially changes the role of the subscapularis, an intact inferior subscapularis assures the joint protection necessary for ROM [22] and the superior subscapularis provides a positive vector force and function as an abductor [23]. Second, preservation of the subscapularis may improve internal rotation. A deficit in internal rotation is common after RSA, and while not well-studied, lack of healing of the subscapularis may partially account for this deficit. Third, if tenotomized or preoperatively torn, the subscapularis should be repaired whenever possible and protected in order to obtain healing as it plays a role in postoperative stability [24] at least in Medial Glenoid/Medial Humerus designs. Fourth, and finally, the SSCS approach allows immediate ROM. Immobilization has been shown to be associated with increased shoulder stiffness [25]. Postoperative immobilization following shoulder arthroplasty has been designed to balance the optimization of healing and prevention of stiffness. A 6-week period of immobilization is typically used to allow the tendon bone interface to

Table 3 Cost by surgical approach evaluated at 3 months post-surgery

\begin{tabular}{|c|c|c|c|c|}
\hline & All prosthesis $(N=35)$ & SSCS approach $(N=17)$ & TDP approach $(N=18)$ & $P$ value \\
\hline Hospitalization stay & $11.9 \pm 10.2$ & $8.2 \pm 6.4$ & $15.2 \pm 11.9$ & 0.04 \\
\hline Hospitalization costs (dollars) & $13,600 \pm 7900$ & $10,500 \pm 5200$ & $16,400 \pm 8700$ & 0.02 \\
\hline Complication rate & $1(3 \%)$ & $0(0 \%)$ & $1(6 \%)$ & 1 \\
\hline Number of outpatient care physical therapy sessions & $14.1 \pm 13.7$ & $15.9 \pm 17.9$ & $12.4 \pm 8.7$ & 0.48 \\
\hline
\end{tabular}

TDP traditional deltopectoral, SSCS subscapularis and deltoid sparing 
progress through the normal healing phases of inflammation, proliferation, and remodeling [26]. After subscapularis repair in anatomic total shoulder arthroplasty, 4 weeks of immobilization lead to higher healing rates [27]. However, with a SSCS approach, immobilization may be avoided since there is no need to obtain subscapularis healing. Such early mobilization likely explains our superior clinical results in the SSCS group at short term. Nevertheless, the results were no different at 1 year.

Complications after RSA are related to etiology [28], prosthetic design $[9,29]$, arm lengthening [30,31], and experience of the surgeon [32]. Traditionally, the rate of short-term complications after RSA is around 20\% [28, 33, 34]. In this case-control series of 35 patients, the rate of short-term complications (3\%) was lower than previously reported. In particular, we did not observe any technical problems with the SSCS approach. While further study with a larger cohort is needed, the early results with deltopectoral approach (with or without subscapularis sparing) are encouraging.

\section{Strengths and limitations}

This prospective case-control study was the first to analyze the impact of a SSCS approach for RSA on cost. We observed substantial economic savings to the system, improved short-term results, and a minimal complication rate that may have the potential to change the standard for approach during RSA. However, there are several limitations that warrant discussion. First, different insurance coverages have been included in the study. The calculation was based on private division fees. Therefore, formal cost analysis was not possible for DRG patients [35] (i.e., patients without a private insurance coverage). Indeed, the cost of RSA for patients with DRG is not dependent of the length of the hospitalization stay. We consequently extrapolated the price regarding the loss of earnings for the hospital. Second, this study represents the learning curve and experience of one surgeon. Results could vary by learning curve and different geographical regions or health care systems. Concern has been expressed about cost savings from small changes in systems and techniques [36]. To date, no study has examined the economic effect of more widespread use of such approach, as it may not deliver significant savings at the macro scale. Effectively, it has not been proven that an anterosuperior approach [37], which involves the splitting of the deltoid muscle to avoid cutting the subscapularis tendon, is associated with lower cost or better functional results [38]. Third, we also recognize that SSCS approach might be challenging in certain cases (i.e., stiff shoulders) and may not be practical or possible in all circumstances. Fourth, our LOS was long. The latter is dependent of many factors, including patient factors (i.e., pain and ability to do ADLs) and health system factors. For example, in our country, our insurance system often imposes a minimum stay which artificially prolongs the LOS. In a recent study, Padegimas et al. demonstrated that LOS at orthopedic specialty hospitals is significantly shorter than at tertiary referral centers [39]. Their findings may be the result not only of fast-track rehabilitation and strict disposition protocols but also of less invasive surgical techniques. The cost-effectiveness of the SSCS approach is now even more apparent in our practice as patients are routinely discharging after only one to two nights in the hospital and no longer require an acute care stay and do not have therapy in the first 6 weeks postoperative. Fifth, due to the limited sample size, some of the comparisons performed might lack statistical power (type II error). Multicenter and prospective investigation will be necessary to determine the role of independent variables such as surgical approach, fast-track surgery, rehabilitation protocols, or health care systems.

\section{Conclusion}

Using a SSCS approach is an option for patients requiring RSA. Overall, LOS is minimized compared to a TDP approach with subscapularis tenotomy. The SSCS approach may provide substantial healthcare cost savings, without increasing complication rate or decreasing patient satisfaction.

\section{Abbreviations}

DRG: Diagnosis-related group; LOS: Length of stay; ROM: Range of motion; RSA: Reverse shoulder arthroplasty; SANE: Single assessment numeric evaluation; SSCS: Subscapularis and deltoid sparing; TDP: Traditional deltopectoral; VAS: Visual analogue scale

\section{Acknowledgements}

Not applicable.

Funding

No funding was received for this study.

Availability of data and materials

Please contact author for data requests.

\section{Authors' contributions}

AL conceived of the study, operated the patients, participated in its design and coordination, and helped to draft the manuscript. JT and AN who were not involved in the surgical procedures analyzed the medical records, operative reports, and radiographs for each patient. PJD and PC helped to draft and to write the manuscript. AS participated in the design of the study and performed the statistical analysis. All authors read and approved the final manuscript

Ethics approval and consent to participate

Ethical committee approval was received from Association des Médecins du Canton de Genève et Société Médicale; protocol 12-26; November 12, 2012. All patients gave informed written consent.

Consent for publication

Not applicable.

\section{Competing interests}

PJD received royalties and is a paid consultant for Arthrex. PC is a paid consultant from Wright and Smith and Nephew and received royalties from Wright, Storz, and Advanced Medical Application. The other authors certify 
that they or any members of their immediate families have no non-financial or financial disclosures (e.g., consultancies, stock ownership, equity interest, patent/licensing arrangements) that might pose a conflict of interest in connection with the submitted article.

\section{Publisher's Note}

Springer Nature remains neutral with regard to jurisdictional claims in published maps and institutional affiliations.

\section{Author details}

${ }^{1}$ Division of Orthopaedics and Trauma Surgery, La Tour Hospital, Rue J.-D. Maillard 3, 1217 Meyrin, Switzerland. 'Faculty of Medicine, University of Geneva, Rue Michel-Servet 1, 1211 Geneva 4, Switzerland. ${ }^{3}$ Division of Orthopaedics and Trauma Surgery, Department of Surgery, Geneva University Hospitals, Rue Gabrielle-Perret-Gentil 4, CH-1211 Geneva 14, Switzerland. ${ }^{4}$ Southern Oregon Orthopedics, Medford, Oregon, USA. ${ }^{5}$ Department of Orthopaedics and Rehabilitation, Oregon Health \& Science University, Portland, Oregon, USA. 'Saint-Grégoire Private Hospital Center, Boulevard Boutière 6, 35768 Saint-Grégoire cedex, France.

Received: 19 April 2017 Accepted: 3 July 2017

\section{Published online: 14 July 2017}

\section{References}

1. Schairer WW, Nwachukwu BU, Lyman S, Craig EV, Gulotta LV. National utilization of reverse total shoulder arthroplasty in the United States. J Shoulder Elbow Surg. 2015;24:91-7.

2. Lädermann A, Lo EY, Schwitzguebel AJ, Yates E. Subscapularis and deltoid preserving anterior approach for reverse shoulder arthroplasty. Orthop Traumatol Surg Res. 2016;102:905-8.

3. Walch $\mathrm{G}$, Wall B. Indication and techniques of revision arthroplasty with a reverse prosthesis. In: Walch G, Boileau P, Mole D, Favard L, Lévigne C, Sirveaux F, editors. Reverse shoulder arthroplasty. Montpellier: Sauramps Medical; 2006. p. 243-6.

4. Boileau P, Walch $G$. The surgical anatomy and osteotomy technique for the humeral head. In: Walch GBP, editor. Shoulder arthroplasty. Berlin: SpringerVerlag; 1999. p. 107-55.

5. Berhouet J, Garaud P, Favard L. Evaluation of the role of glenosphere design and humeral component retroversion in avoiding scapular notching during reverse shoulder arthroplasty. J Shoulder Elbow Surg. 2014;23:151-8.

6. Gulotta LV, Choi D, Marinello P, Knutson Z, Lipman J, Wright T, Cordasco FA, Craig EV, Warren RF. Humeral component retroversion in reverse total shoulder arthroplasty: a biomechanical study. J Shoulder Elbow Surg. 2012; 21:1121-7.

7. Stephenson DR, Oh JH, McGarry MH, Rick Hatch 3rd GF, Lee TQ. Effect of humeral component version on impingement in reverse total shoulder arthroplasty. J Shoulder Elbow Surg. 2011;20:652-8.

8. Lädermann A, Gueorguiev B, Charbonnier C, Stimec BV, Fasel JH, Zderic I, Hagen J, Walch G. Scapular notching on kinematic simulated range of motion after reverse shoulder arthroplasty is not the result of impingement in adduction. Medicine (Baltimore). 2015;94:e1615.

9. Lädermann A, Denard PJ, Boileau P, Farron A, Deransart P, Terrier A, Ston J, Walch G. Effect of humeral stem design on humeral position and range of motion in reverse shoulder arthroplasty. Int Orthop. 2015;39:2205-13.

10. Lovallo J, Helming J, Jafari SM, Owusu-Forfie A, Donovan S, Minnock C, Adib F. Intraoperative intra-articular injection of gentamicin: will it decrease the risk of infection in total shoulder arthroplasty? J Shoulder Elbow Surg. 2014; 23:1272-6

11. Liotard J. Painful shoulder rehabilitation: how to do it simple. Revue du rhumatisme monographies. 2010;77(3):239-45.

12. Constant $\mathrm{CR}$, Murley $\mathrm{AH}$. A clinical method of functional assessment of the shoulder. Clin Orthop Relat Res. 1987;214:160-4.

13. Menendez ME, Baker DK, Fryberger CT, Ponce BA. Predictors of extended length of stay after elective shoulder arthroplasty. J Shoulder Elbow Surg. 2015;24:1527-33

14. Bachman D, Nyland J, Krupp R. Reverse-total shoulder arthroplasty costeffectiveness: a quality-adjusted life years comparison with total hip arthroplasty. World J Orthop. 2016;7:123-7.

15. Garcia GH, Fu MC, Dines DM, Craig EV, Gulotta LV. Malnutrition: a marker for increased complications, mortality, and length of stay after total shoulder arthroplasty. J Shoulder Elbow Surg. 2016;25:193-200.
16. Sivasundaram L, Heckmann N, Pannell WC, Alluri RK, Omid R, Hatch 3rd GF. Preoperative risk factors for discharge to a postacute care facility after shoulder arthroplasty. J Shoulder Elbow Surg. 2016;25:201-6.

17. Hammond JW, Queale WS, Kim TK, McFarland EG. Surgeon experience and clinical and economic outcomes for shoulder arthroplasty. J Bone Joint Surg Am. 2003;85-A:2318-24.

18. Hassan M, Tuckman HP, Patrick RH, Kountz DS, Kohn JL. Cost of hospitalacquired infection. Hosp Top. 2010;88:82-9.

19. Xu S, Baker DK, Woods JC, Brabston 3rd EW, Ponce BA. Risk factors for early readmission after anatomical or reverse total shoulder arthroplasty. Am J Orthop (Belle Mead NJ). 2016:45:E386-92.

20. Husted H, Holm G, Jacobsen S. Predictors of length of stay and patient satisfaction after hip and knee replacement surgery: fast-track experience in 712 patients. Acta Orthop. 2008;79:168-73.

21. Collin P, Matsumura N, Lädermann A, Denard PJ, Walch G. Relationship between massive chronic rotator cuff tear pattern and loss of active shoulder range of motion. J Shoulder Elbow Surg. 2014;23:1195-202.

22. Collin P, Lädermann A, Le Bourg M, Walch G. Subscapularis minor-an analogue of the Teres minor? Orthop Traumatol Surg Res. 2013;99:S255-8.

23. Gulotta LV, Choi D, Marinello P, Wright T, Cordasco FA, Craig EV, Warren RF. Anterior deltoid deficiency in reverse total shoulder replacement: a biomechanical study with cadavers. J Bone Joint Surg (Br). 2012;94:1666-9.

24. Edwards TB, Williams MD, Labriola JE, Elkousy HA, Gartsman GM, O'Conno DP. Subscapularis insufficiency and the risk of shoulder dislocation after reverse shoulder arthroplasty. J Shoulder Elbow Surg. 2009;18:892-6.

25. Sarver JJ, Peltz CD, Dourte L, Reddy S, Williams GR, Soslowsky LJ. After rotator cuff repair, stiffness-but not the loss in range of motion-increased transiently for immobilized shoulders in a rat model. J Shoulder Elbow Surg. 2008;17:108S-13.

26. Millett PJ, Wilcox 3rd RB, O'Holleran JD, Warner JJ. Rehabilitation of the rotator cuff: an evaluation-based approach. J Am Acad Orthop Surg. 2006; 14:599-609.

27. Denard PJ, Lädermann A. Immediate versus delayed passive range of motion following total shoulder arthroplasty. J Shoulder Elbow Surg. 2016; 25:1918-24.

28. Wall B, Nove-Josserand L, O'Connor DP, Edwards TB, Walch G. Reverse total shoulder arthroplasty: a review of results according to etiology. J Bone Joint Surg Am. 2007;89:1476-85.

29. Farshad M, Gerber C. Reverse total shoulder arthroplasty — from the most to the least common complication. Int Orthop. 2010;34:1075-82.

30. Lädermann A, Edwards TB, Walch G. Arm lengthening after reverse shoulder arthroplasty: a review. Int Orthop. 2014;38:991-1000.

31. Lädermann A, Lubbeke A, Melis B, Stern R, Christofilopoulos P, Bacle G, Walch G. Prevalence of neurologic lesions after total shoulder arthroplasty. J Bone Joint Surg Am. 2011;93:1288-93.

32. Walch G, Bacle G, Lädermann A, Nove-Josserand L, Smithers CJ. Do the indications, results, and complications of reverse shoulder arthroplasty change with surgeon's experience? J Shoulder Elbow Surg. 2012;21:1470-7.

33. Clark JC, Ritchie J, Song FS, Kissenberth MJ, Tolan SJ, Hart ND, Hawkins RJ. Complication rates, dislocation, pain, and postoperative range of motion after reverse shoulder arthroplasty in patients with and without repair of the subscapularis. J Shoulder Elbow Surg. 2012;21:36-41.

34. Mulieri P, Dunning P, Klein S, Pupello D, Frankle M. Reverse shoulder arthroplasty for the treatment of irreparable rotator cuff tear without glenohumeral arthritis. J Bone Joint Surg Am. 2010;92:2544-56.

35. Busse R, Geissler A, Aaviksoo A, Cots F, Hakkinen U, Kobel C, Mateus C, Or Z, O'Reilly J, Serden L, et al. Diagnosis related groups in Europe: moving towards transparency, efficiency, and quality in hospitals? BMJ. 2013;346: f3197.

36. Marshall M, Ovretveit J. Can we save money by improving quality? BMJ Qual Saf. 2011:20:293-6.

37. Mole D, Wein F, Dezaly C, Valenti P, Sirveaux F. Surgical technique: the anterosuperior approach for reverse shoulder arthroplasty. Clin Orthop Relat Res. 2011:469:2461-8.

38. Lädermann A, Lubbeke A, Collin P, Edwards TB, Sirveaux F, Walch G. Influence of surgical approach on functional outcome in reverse shoulder arthroplasty. Orthop Traumatol Surg Res. 2011;97:579-82.

39. Padegimas EM, Zmistowski BM, Clyde CT, Restrepo C, Abboud JA, Lazarus MD, Ramsey ML, Williams GR, Namdari S. Length of stay after shoulder arthroplasty-the effect of an orthopedic specialty hospital. J Shoulder Elbow Surg. 2016:25:1404-11. 